Article

\title{
La conservation de la biodiversité peut-elle être une source d'inspiration pour le patrimoine architectural ?
}

\author{
Walid Hamma ${ }^{1}$, Florentina-Cristina Merciu ${ }^{2}$, Alexandru-Ionuț Petrişor ${ }^{3}{ }^{凶}$, \\ Andreea-Loreta Cercleux ${ }^{4}$
}

\author{
${ }^{1}$ Université de Tlemcen, Département d'Architecture, Algérie \\ ${ }_{2}^{2}$ Université de Bucarest, Centre Interdisciplinaire des Études Avancées sur la Dynamique Territoriale, Roumanie \\ ${ }^{3}$ Université d'Architecture et d'Urbanisme «Ion Mincu » de Bucarest, Roumanie \\ ${ }^{4}$ Université de Bucarest, Faculté de Géographie, Roumanie
}

\begin{abstract}
Résumé. L'article présente plusieurs principes utilisés dans la conservation biologique (par les aires naturelles protégées) qui servent comme sources d'inspiration pour la conservation du patrimoine architectural. Sous l'influence du Club de Rome et sa théorie de " croissance zéro ", l'idée de la conservation a été aperçue comme la préservation ou la conservation stricte, dans un état intact, incompatible avec le développement et, cependant, utopique. La nouvelle vue agit pour la maintenance dans un «état favorable » qui permet le développement et n'affecte pas la capacité de soutien des systèmes écologiques. Les principes examinés dans l'étude sont la conservation fonctionnelle (dans les limites des capacités de charge) permettant une gestion progressive des activités de conservation, le choix des bâtiments représentatifs à protéger, l'implication des communautés locales et le soutien international. L'idée centrale de l'article est que le soutien de la communauté locale est très important pour la conservation. Dans ce contexte, les principes de la conservation biologique - conservation fonctionnelle, gestion différentiée, représentativité, soutien local et international, zonage interne, coopération nationale et internationale - sont également applicables à la conservation du patrimoine architectural.

Mots clés : patrimoine, planification, participation publique, soutien local, durabilité.
\end{abstract}

\section{CORRESPONDANCE :}

$\triangle$ Str. Academiei no 18-20, secteur 1, CP 01001, Bucarest, Roumanie (A.-I. P.).

\@hammawalid06@hotmail.com(W.H), alexandru_petrisor@ yahoo.com (A.-I. P.), krysten1009@yahoo.com (F.-C. M.), loretacepoiu@yahoo.com (A.-L. C.)
HISTOIRE DE L'ARTICLE :

Reçu : 25 Septembre 2017

Reçu en forme révisée : 23 Juillet 2018

Accepté : 24 Juillet 2018

Disponible en ligne : 10 Octobre 2018 


\section{Introduction}

Dans le sens le plus large, l'héritage culturel est, en même temps, un produit et un processus qui offre aux sociétés une multitude de ressources héritées du passé, crées dans le présent pour le bénéfice des prochaines générations (UNESCO, 2014:130). La diversité de l'héritage culturel dans le monde contemporain repose sur la richesse culturelle des sociétés à multiples facettes. Le patrimoine architectural construit se trouve parmi les plus importants éléments composant le patrimoine cultural tangible. L'importance croissante du patrimoine culturel est liée à son caractère multivalent (historique, architectural, symbolique, spirituel, éducationnel, pérenne, esthétique, scientifique, commémoratif - quelques dimensions se superposent ou sont en compétition etc.). Pour ces raisons, celui-ci joue un rôle important dans la société contemporaine (Frey, 1997; Throsby, 2002; DYNAMO Transnational Group, 2006; Vecco, 2010; Greffe, 2011; Ferretti et al., 2014; Merciu et al., 2014). Les valeurs de l'héritage culturel justifient la nécessité de le préserver et capitaliser par différentes groups et classes sociales (Maroevic, 1998; Mason, 2002; Graz, 2011).

L'héritage culturel est de plus en plus menacé avec la dégradation et la destruction par quelques facteurs de risque issus des causes environnementales naturelles (comme les tremblements de terre, les glissements du terrain, les volcans, les inondations, les avalanches, les changements climatiques ou la dynamique côtière) ou des activités humaines (la pression des touristes et utilisateurs, l'urbanisation sans planification, les projets de développement destructifs, les vols, le vandalisme, les conflits, la pollution de l'air et visuelle) (Mourato et Mazzanti, 2002; Vicente et al., 2006; UNESCO, 2008; Sabbioni et al., 2008; Grasso \& Maugeri, 2009; Pessina \& Meroni, 2009; ICOMOS, 2009; Pagini et al., 2012; Stephenson et D'Ayala, 2014; Cercleux, 2015; Cercleux et al., 2016; Nocca, 2017).

La conservation du patrimoine produit aussi des valeurs économiques, définies comme un bien public qui reflète les décisions collectives plus qu'individuelles et qui ne sont pas saisies par les mesures des prix du marché. Beaucoup de biens culturels ne sont pas sujet de transaction sur le marché, ayant un « prix zéro ». Ceux-ci sont appelés des ressources culturelles non-commerciales et évalués par la société d'une manière qui n'est pas traduite dans aucun prix de marché (Mourato et Mazzanti, 2002). Il y a une distinction importante entre quels valeurs peuvent être représentées d'une manière légitime du point de vue du prix (valeurs privées qui font le sujet des transactions sur le marché) et quels facteurs déterminent les décisions d'octroyer les ressources, publiques ou collectives (Mason, 2002).

La conservation de l'héritage culturel est une préoccupation internationale permanente. Il y a des politiques pour sa préservation, protection et maintenance, ayant comme but l'augmentation de son importance et sa transmission aux 
générations futures. Cependant, les bénéfices économiques résultant par sa conservation excèdent les coûts. La conservation est un investissement sage (Ruijgrok, 2006) réalisé par des travaux de rénovation et maintenance des bâtiments de patrimoine, qui représentent plus d'un quart de la valeur de l'industrie européenne de construction (European Commission, 2015).

Les impacts multidimensionnels (économique, social et environnemental) des investissements dans l'héritage culturel reflètent sa capacité de contribuer à la croissance de la productivité locale totale, d'améliorer le bien-être des résidents et d'attirer du financement des secteurs publique, privé ou publique-privé. Cette perspective multidimensionnelle implique une approche systémique et intégré, nécessitant des outils d'évaluation (méthodes, indicateurs, etc.) capables de comparer les coûts de la conservation du passé avec les bénéfices du changement (Cercleux et al., 2012; Nocca, 2017). L'évidence empirique des bénéfices multidimensionnels de la conservation de l'héritage culturel (et sa contribution au développement durable) manque encore, créant le besoin d'une approche holistique (Nocca, 2017).

D'un point de vue écologique, la restauration de l'héritage bâti est une " procédure d'intervention minimale " avec one fonction d'avant-garde dans la démonstration d'un type de développement durable, économisant l'utilisation des ressources culturelles et historiques non-renouvelables, protégeant les ressources non-renouvelables et la matière première contre la supra-exploitation et évitant la supra-abondance des nouveaux bâtiments. L'utilisation et le changement continue du patrimoine bâti implique toujours une réduction de la consommation des matériaux de construction (Haspel, 2011:910).

La science de la conservation représente une approche transdisciplinaire (Heritage et Golfomitsou, 2015), menée à conserver également les objets appartenant à l'héritage culturel et à la nature (Sandu, 2010) et à les réintégrer du point de vue structurelle et fonctionnelle (Cercleux et al., 2012; Merciu et al., 2016), de l'ambiant et culturel (Sandu et al., 2016). Dans la nomenclature de la science de la conservation de l'héritage culturel, la conservation suppose la perpétuation du patrimoine, la préservation signifie la prévention et les interventions sur le patrimoine et la restauration fait référence à la consolidation et la réintégration du patrimoine (Sandu et al., 2016). En effet, il y a huit aspects lucratifs de la conservation : (1) découvrir, (2) classifier et évaluer, (3) investiguer, (4) préserver d'une manière passive (climatiser), (5) préserver d'une manière active (traiter), (6) restaurer, (7) montrer, valoriser et thésauriser et (8) protéger et maintenir (Sandu et al., 2016).

La réutilisation des bâtiments historiques est une décision complexe (Ferretti et al., 2014) en raison de la multitude de facteurs qui doivent être pris en compte dans la détermination d'une exploitation optimale: la nécessité de remplir plusieurs objectifs (conservation / restauration le cas échéant, mise en valeur), le régime de propriété des bâtiments de patrimoine et les différents acteurs participants (Merciu et 
al., 2016). La problématique du processus de réutilisation des bâtiments de patrimoine est représentée par l'évaluation de sa durabilité, c'est à dire de la compatibilité de la solution de conversion avec les valences des monuments historiques, en assurant l'intégrité architecturale des bâtiments et que la nouvelle fonction représente leur réutilisation optimale (Elsorady, 2014).

Même s'il y a encore des débats concernant notamment les enjeux, le concept de « conservation de la biodiversité » est présent sur les agendas politiques locaux, régionaux et nationaux dans le monde entier, étant imposé par la Convention signé à Rio de Janeiro en 1992 et les Agendas 21 dérivées. Malgré les tentatives d'application qui n'ont pas été toujours positives, l'expérience accumulée dans ces 25 ans peut fournir des leçons pour les autres domaines. L'approche du patrimoine, soit-il naturel ou bâti, est commune - voir les conventions de Paris (1972), Torremolinos (1983), Granada (1985), Rio de Janeiro (1992), Florence (2000), Faro (2005) etc. (Naveh, 1994, 1998 ; Petrişor, 2011b). Pour cette raison, l'article présente la possibilité de transférer quelques principes utilisés dans la conservation de la biodiversité vers le patrimoine architectural (bâti).

L'importance actuelle du patrimoine culturel européen est démontrée dans dix perspectives : (1) contribution à l'attractivité des régions et villes ; (2) contribution à l'identité nationale ou régionale ; (3) création d'emplois ; (4) source de créativité et innovation ; (5) génération du profit et retour des investissements ; (6) catalyser la régénération durable ; (7) solution contre les changements climatiques ; (8) contribution à la qualité de vie ; (9) encouragement de l'éducation et de la formation continue ; (10) construction du capital social et génération de la cohésion sociale (CHCfE Consortium, 2015).

Pendant les derniers ans, le processus de conservation a été analysé dans une perspective multidisciplinaire, impliquant la coopération des spécialistes appartenant aux multiples disciplines (Petrişor, 2013). Le domaine de la protection de l'environnement a une longue tradition de consultation d'un large spectre des parties intéressées. Les approches utilisées dans la protection de l'environnement sont souvent soutenues comme exemples qui seront émulées pour le patrimoine (de la Torre et Mason, 2002).

\section{Le grand débat : développement ou conservation ?}

Du point de vue théorique, le développement durable est « un développement qui répond aux besoins du présent sans compromettre la capacité des générations futures de répondre aux leurs » (Brundtland, 1987). Si le concept « besoin » fait la liaison avec l'utilisation des ressources dans les limites de la capacité du support écologique, en pratique le développement durable agit pour mettre en cohérence les attentes économiques et sociales par rapport au territoire avec ses fonctions écologiques et 
culturelles, améliorant la qualité de vie des générations présentes et futures et en créant des communautés durables, capables de gérer et d'utiliser les ressources de manière efficace et d'exploiter le potentiel d'innovation écologique et sociale de l'économie, en garantissant la prospérité, la protection de l'environnement et la cohésion sociale (Collignon, 2009). Ces définitions montrent que le développement durable et la conservation ne sont pas opposés (Nasser, 2003). Si le développement durable assume une responsabilité pour les futures générations, la conservation permet de garder pour ceux-ci une partie du patrimoine actuel (Hoag et Skold, 1996 ; Holling, 2000; Marques, 2001; Rodwell, 2003, 2007; Cercleux et al., 2012; Hamma, 2017a, b; Bouroumi et al., 2017; Hamma et al., 2017).

Les principes du développement durable ont prouvé d'être utiles, efficaces et robustes pas seulement pour la conservation de l'environnement et pour promouvoir la sensibilité écologique dans le domaine du développement, mais de plus en plus dans le domaine du développement urbain. La durabilité a été proposée comme but et aussi comme guide pour les politiques du patrimoine (English Heritage, 1997; Mason, 2002).

Throsby (2002) a proposé un set de principes de durabilité qui puissent constituer la base d'un set utile des normes de décision dans la planification de la conservation (génération de bénéfices tangibles et non-tangibles, équité intergénérationnelle, équité intragénérationnelle, maintenance de la diversité, le principe de la prudence, la reconnaissance de l'interdépendance). Les principes sont construits utilisant la notion de durabilité développée dans le domaine de la conservation écologique et développement économique et adaptée à la notion de capital culturel (ressources de patrimoine), qui dans la vision de Throsby avec analogue au capital naturel. La métaphore du « capital » est, potentiellement, très utile. Celle-ci reflète le besoin d'investir dans le patrimoine, l'investissement contribuant à la création de plusieurs bénéfices (salaires, emplois etc.).

La notion de la durabilité est conforme aux principes qui constituent la base de la planification de la conservation, qui repose sur les valeurs par le fait qu'elle adopte une vision globale des ressources (dans ce cas, les ressources culturelles) et leur contexte, en s'alignant à l'objectif de prendre en considération la plus large gamme de valeurs de patrimoine. Cela s'occupe directement de la problématique de prendre les décisions dans le présent, mais aussi pour à long terme - essentiel pour la reconnaissance du rôle du patrimoine comme héritage qui sera géré et transféré aux générations futures.

\section{Une nouvelle vue de la conservation}

Sous l'influence du Club de Rome et de sa théorie de «croissance zéro », l'idée de la 
conservation a été aperçue par la préservation ou la conservation stricte, dans un état intact, incompatible avec le développement et, cependant, utopique. La nouvelle vue agit pour la maintenance dans un « état favorable » qui permet le développement, étant donné que n'affecte pas la capacité de soutien des systèmes écologiques (Petrişor, 2011c). En effet, cette approche permet une évaluation fonctionnelle, plus importante que l'approche structurelle.

Le débat existe aussi dans l'architecture. Dennis Rodwell, architecte spécialisé dans la conservation, écrivait en 1992 : «Je vois la conservation marginalisée, dominée par les historiens de l'architecture et sans aucune connexion avec les fonctions des bâtiments et les villes historiques - sauf quand ils deviennent une scène historique. Je ne trouve aucune motivation dans les conditions et le cadre quotidien des citoyens ». Nous pouvons remarquer la même idée, suggérant le besoin d'une approche fonctionnelle (Rodwell, 1992).

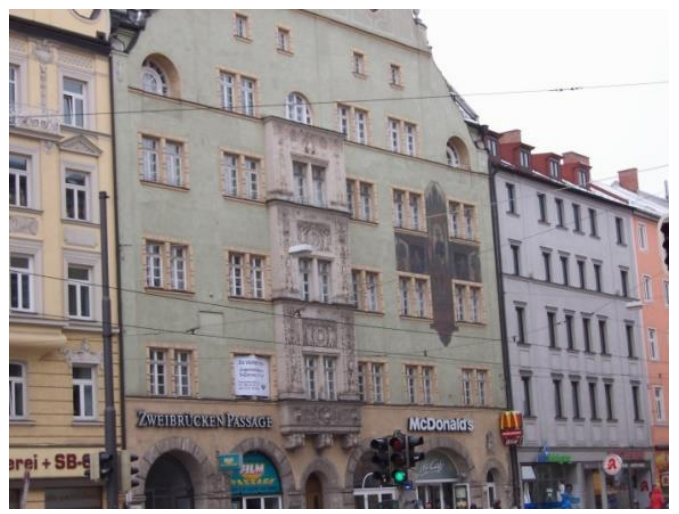

Figure 1: Préservation d'une façade historique à Munich, Allemagne : malgré le fait que la fonction d'un bâtiment a changé radicalement et le caractère historique de la zone est perdu. Photo prise par Alexandru-Ionuţ Petrişor

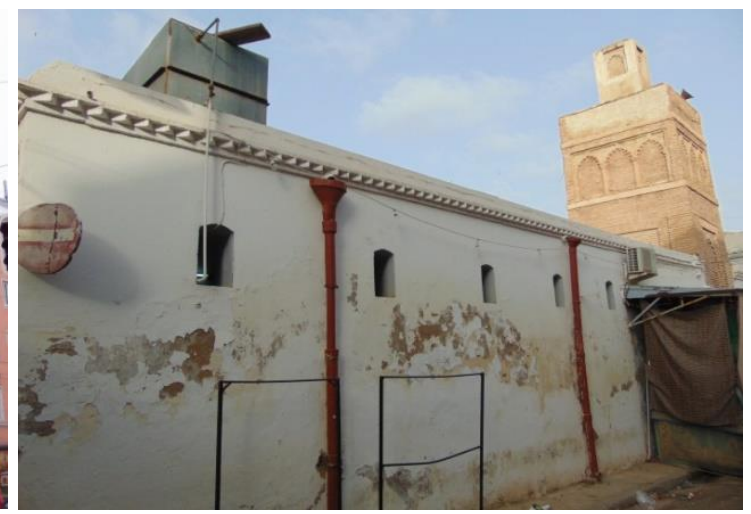

Figure 2: Restauration de mosquée Sidi El Benna du 14ème siècle à Tlemcen, Algérie. Nous pouvons voir que l'approche non scientifique a défiguré le monument par le blanchissement du minaret, les climatiseurs et réservoirs installés sur le toit, les systèmes modernes d'évacuation des eaux et les constructions parasitaires ajoutées au monument. Photo prise par Alexandru-Ionuţ Petrişor

Deux mauvais exemples viennent de l'Allemagne et de l'Algérie. Le premier est la manière de préserver une façade historique, malgré le fait que la fonction d'un bâtiment a changé radicalement et le caractère historique de la zone est perdu - voir la photo en Figure 1, prise à Munich, Allemagne. Le deuxième est la restauration menée pour conserver la mosquée de Sidi El Benna de Tlemcen, Algérie (14ème siècle) ; le caractère historique est perdu par le blanchissement du minaret, les climatiseurs et réservoirs installés sur le toit, les systèmes modernes d'évacuation des eaux pluviales et les constructions parasitaires ajoutées au monument (Figure 2). 
Deux bons exemples viennent de Roumanie et sont présentés dans les deux photos qui suivent : dans la Figure 3 - la restauration de la citadelle Vauban d'Alba Iulia, finalisée et dans la Figure 4 - la restauration de la citadelle de Râşnov (Rosenau), en cours de finalisation. La citadelle médiévale d'Alba Iulia a été sous l'administration de l'armée pendant la période communiste (avant 1989). L'armée n'a pas fait des investissements dans la restauration des murs, mais a bâti des constructions parasitaires qui ont été ensuite démolies (Photo A). La restauration de la citadelle a été financée avec des fonds européens. Un élément clé est la formation d'une «garde autrichienne » qui attire les touristes (Photo B), mai aussi le mobilier urbain (Photo C). Une bonne connexion avec la ville est assurée pendant le jour et la nuit. La citadelle de Râşnov est un exemple de citadelle fortifiée paysanne qui, malheureusement, n'a pas bénéficié des fonds de restauration et les travaux de réparation se déroulent lentement. Nous remarquons néanmoins une préoccupation pour intégrer la citadelle dans la ville urbaine pendant le jour et la nuit (Photos A et B), y compris par des activités "médiévales » avec des personnages historiques (Photos C-E).

Cette vue implique une connexion entre la restauration et la conservation. Par analogie avec la conservation et la restauration des systèmes biologiques, la restauration est une partie de la conservation, menée afin de sauvegarder pour les prochaines générations une structure fonctionnelle. La conservation s'adresse à un composant d'un système, mais la restauration prenne en considération le système entier. Les principes essentiels de la restauration incluent le traitement des causes de la dégradation et la compensation des processus et mécanismes perdus.

Un problème similaire est le zonage interne. Si une aire naturelle protégée peut avoir une organisation spatiale interne correspondante aux différentes catégories de gestion, il est difficile de faire la même chose avec un bâtiment. Cependant, une portion historique urbaine permet l'application du principe. L'idée est d'avoir une gestion moins restrictive en s'écartant d'un «centre » qui doit être strictement protégé, pour faire la transition de conservation au développement (Petrişor, 2011c). Ce principe est connecté avec le précèdent : on peut éviter d'avoir une " fossile » historique dans une région complètement nouvelle, sans aucune liaison entre les deux, en assurant une transition graduelle.

\section{Conservation des échantillons représentatifs avec le soutien local et le financement international}

Quelle est, dans ce cas, la capacité de soutien ? Celle-ci agit sur l'interférence avec les activités d'aujourd'hui des locaux. Il faudra conserver sans affecter plus que nécessaire la vie locale ; cependant, si le but final est d'agrandir l'attractivité locale par le tourisme, il faudra limiter le tourisme agressif et celui qui s'interfère avec la vie 
locale.

Dans la conservation de la biodiversité, les grandes aires naturelles protégées - parcs nationaux ou paysages protégés - ont l'objectif d'inclure des échantillons représentatifs pour l'espace biogéographique national ou régional (Petrişor, 2011c). L'application de ce principe dans la conservation architecturale agit pour trouver l'équilibre - c'est-à-dire, les bâtiments qui sont représentatifs sans aucun doute pour n'importe quelle période ou n'importe quel style architectural.

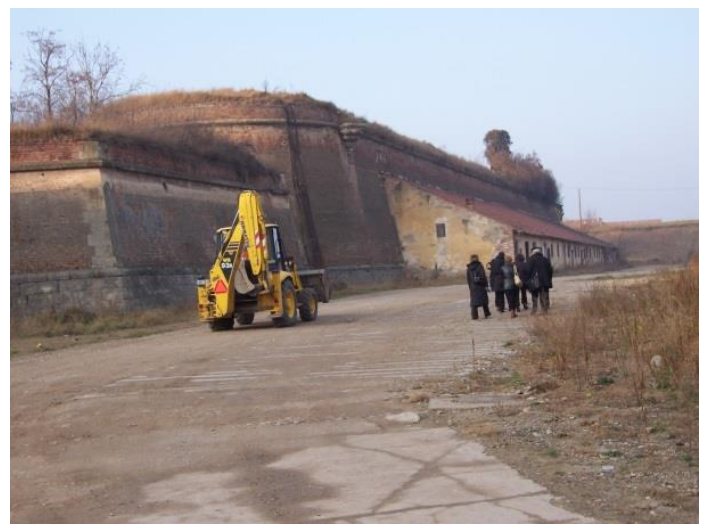

A

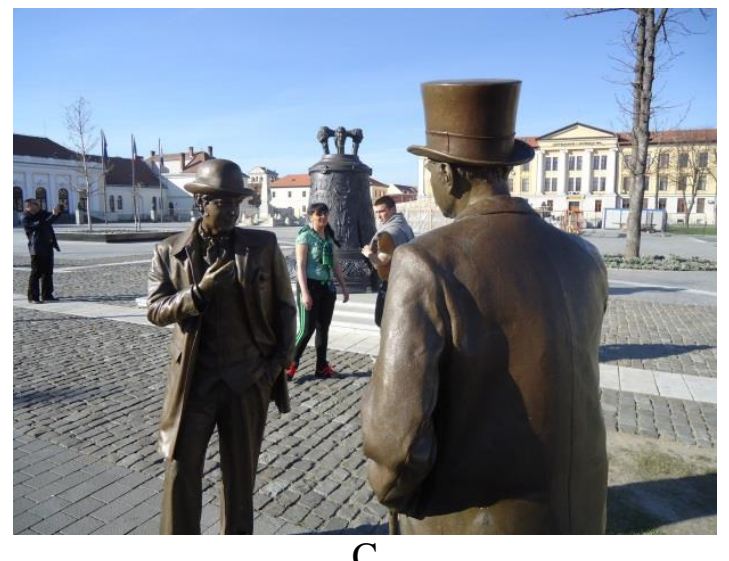

$\mathrm{C}$

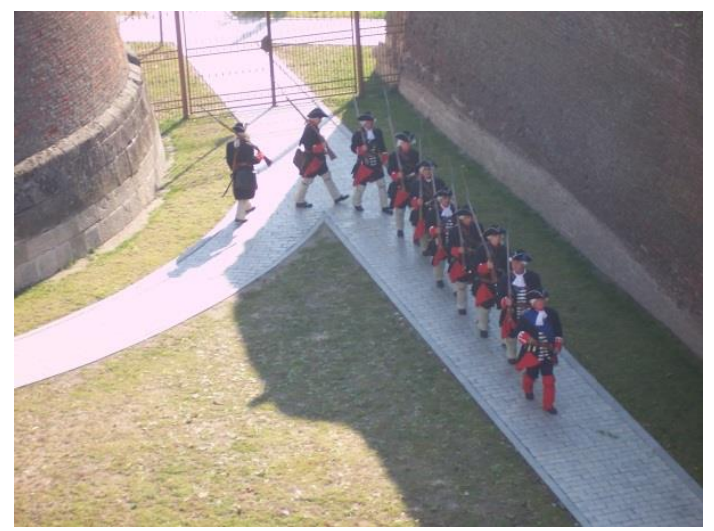

B

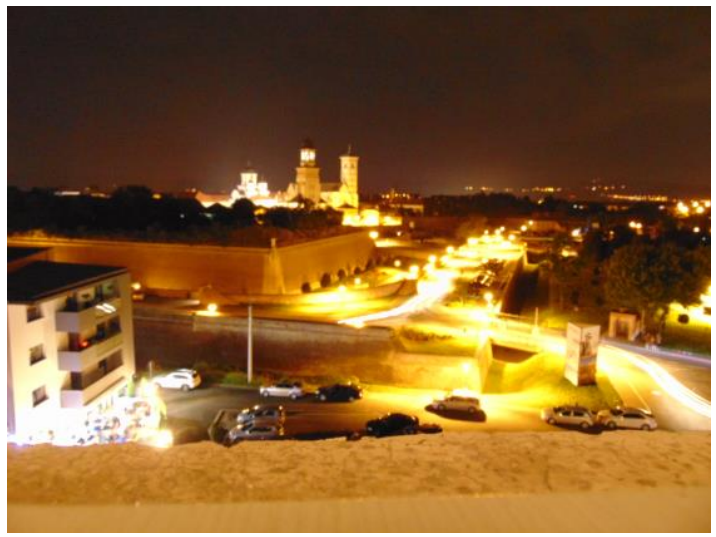

$\mathrm{D}$

Figure 3 : Aspects de la restauration de la citadelle Vauban d'Alba Iulia, Roumanie. Photos prises par Alexandru-Ionuţ Petrişor. A - Début des travaux de restauration, 2006 ; pendant le processus, les constructions parasitaires (élevées par l'armée) ont été démolies, pour libérer les murs de la citadelle Vauban ; B - Parmi les attractions, la garde autrichienne, formée par des volontaires, offre un spectacle incluant le tour de la citadelle et quelques fois les tirs de cannons ; C - le mobilier urbain, faisant une connexion avec l'histoire, attire les touristes et les citoyens de la ville pendant le jour et la nuit dans la citadelle ; D - la citadelle pendant la nuit.

Le soutien local reste essentiel dans la conservation de la biodiversité pour l'élaboration et la mise en exécution du plan de gestion, notamment pour ceux qui ont une importance internationale (Berkes et al., 1995; Petrişor, 2011c). En général, les 
aires naturelles protégées imposées par les conventions internationales peuvent générer des conflits avec la population locale (Nelson et Vertinsky, 2005). Cependant, le soutien international est également important par la possibilité de financement des projets de conservation et de créer des « groupes de soutien » ou des réseaux d'aires naturelles protégées, facilitant le changement des bonnes pratiques.

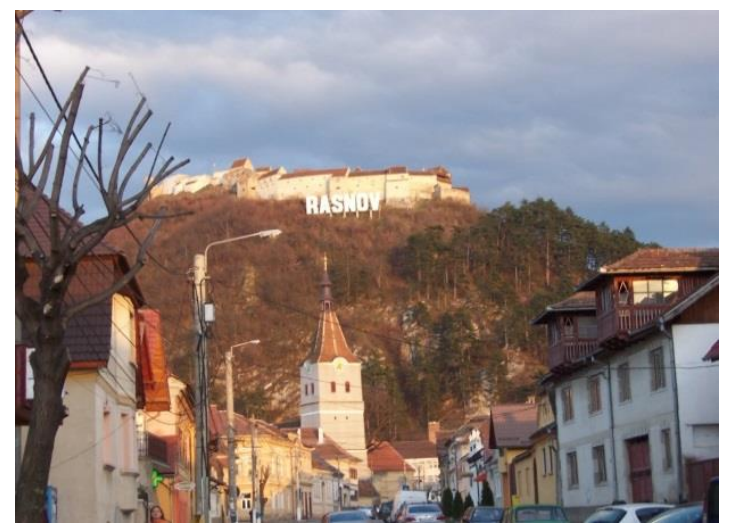

A

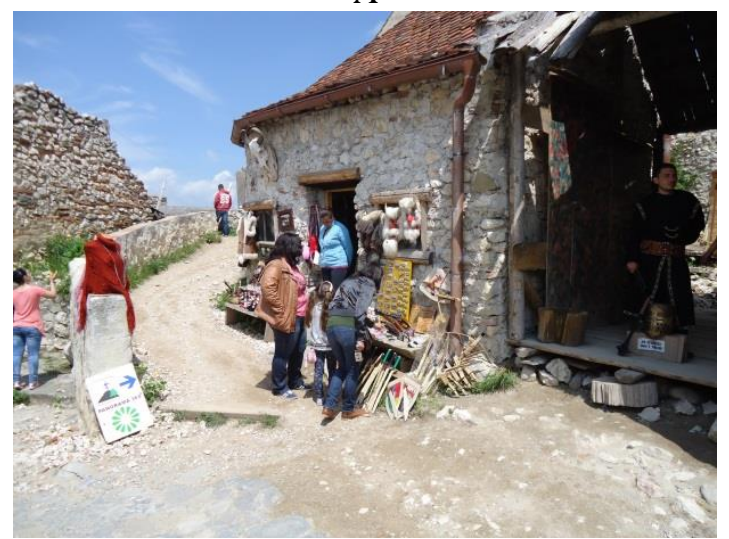

C

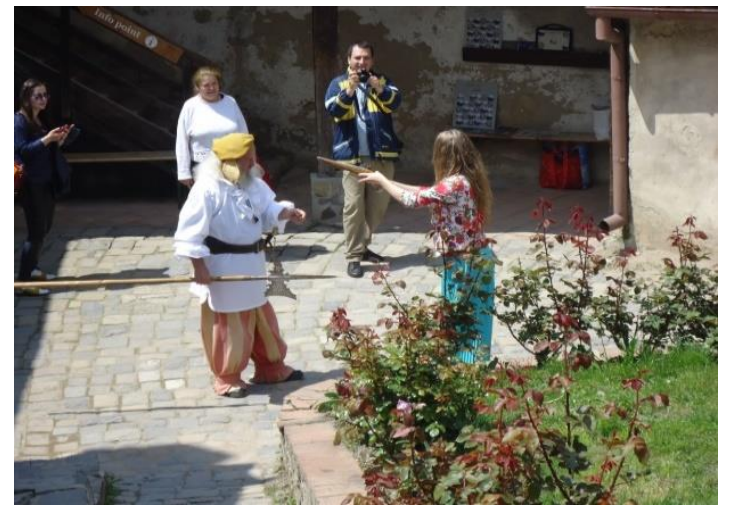

$\mathrm{E}$

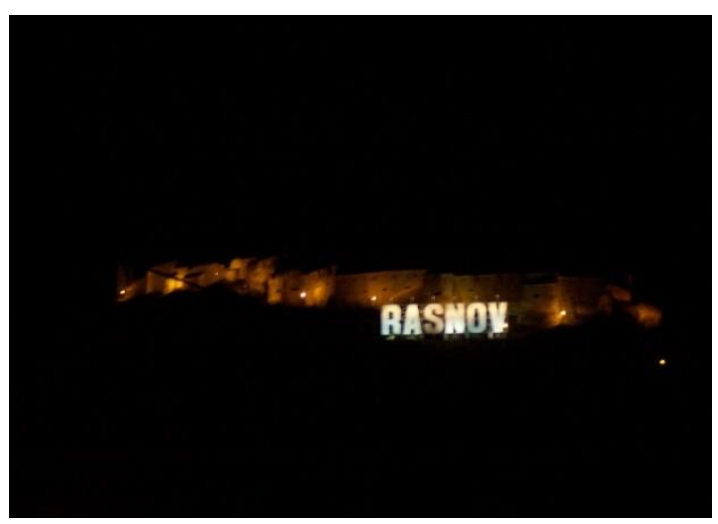

B

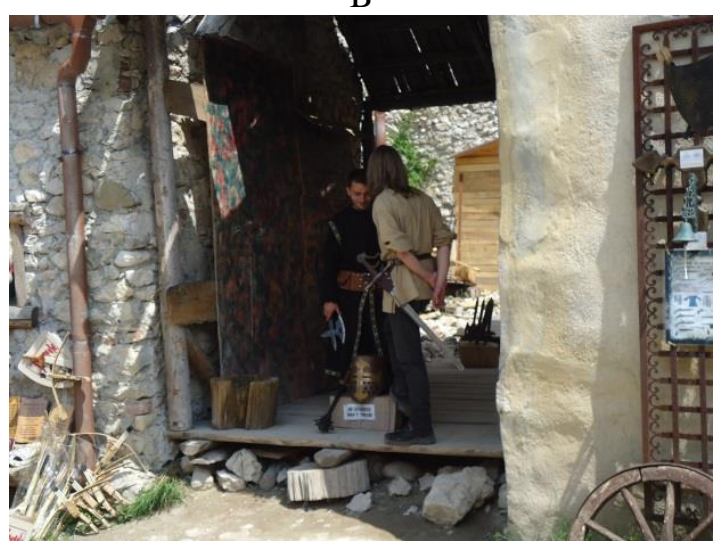

D

A et $B$ - la citadelle qui fait partie de la vie urbaine pendant le jour et la nuit; $\mathrm{C}-\mathrm{E}$ - les personnages historiques sont des volontaires, en majorité. Les associations « médiévales » offrent un spectacle d'armes, des démonstrations demandant la participation des visiteurs, présentent aussi des produits traditionnels, quelquefois avec une spécificité médiévale. Malheureusement, les fonds ne sont pas suffisants pour la restauration des bâtiments (voir Photos C et D).

Figure 3: Aspects de la restauration de la citadelle paysanne de Râşnov, Roumanie. Photos prises par Alexandru-Ionuţ Petrişor 
Encore une fois, l'application de ces principes demande de trouver la meilleure formule de négociation avec la population locale; donné le caractère participatif de l'urbanisme (Lacaze, 1990), le processus de planification participative donne la possibilité d'obtenir le consensus par le débat publique.

\section{Zonage interne}

Dans les aires naturelles protégées, la conservation est faite par un zonage externe, qui permet un tel «dosage » de l'intensité des activités humaines. D'habitude le nucleus strictement protégé se trouve à l'intérieur d'une zone tampon, incorporée dans une zone de développement durable. Le zonage est plus visible dans les grandes aires naturelles protégées comme les parcs (nationaux ou naturels équivalent aux "paysages protégés », géo-parcs) et les réservations de la biodiversité (Petrişor, 2011c). Pour les monuments, il y a des limites de protection et d'autres réglementations concernant la construction des nouveaux bâtiments dans leur proximité (hauteur, couleur, matériaux, fonctions etc.), établis par les plans d'urbanisme. Dans la Figure 5 est représenté le nouveau McDonald's dans une zone historique de Columbia, Caroline du Sud, États-Unis. La nouvelle construction n'a pas l'aspect caractéristique d'un McDonald's, mais elle a l'architecture traditionnelle de la région (matériaux et couleurs). Le deuxième faux étage est bâti pour s'encadrer à la hauteur de la zone.

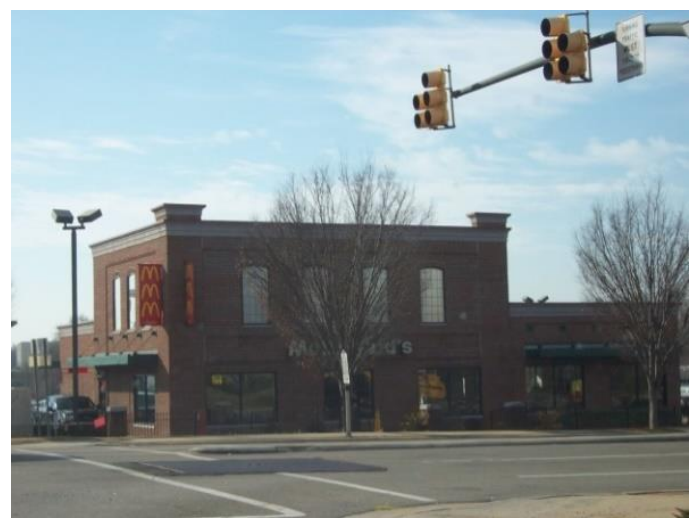

Figure 4: Nouveau McDonald's dans une zone historique de Columbia, Caroline du Sud, États-Unis. La nouvelle construction n'a pas l'aspect caractéristique d'un McDonald's, mais a l'architecture traditionnelle de la région (matériaux et couleurs). Le deuxième faux étage est bâti pour s'encadrer à la hauteur de la zone. Photo prise par Alexandru-Ionuţ Petrişor

\section{Coopération nationale et internationale}

La coopération nationale et internationale est essentielle pour l'échange de bonnes pratiques, mais principalement pour établir les critères pour octroyer le statut de protection et (peut-être le plus important) les mécanismes de financement. Dans le 
cas des aires naturelles protégées, la plupart des aires d'un intérêt plus que national forme des réseaux établis par les conventions internationales (Convention pour la protection de la biodiversité, Convention du paysage, Convention de Ramsar, Convention pour la protection du patrimoine naturel et culturel) ou européennes (Convention pour la protection de l'habitat, Convention pour la protection des oiseaux aquatiques, Convention pour les géo-parcs). La protection des monuments est faite aussi par des conventions internationales (Conventions de La Valetta, Faro, Namur, Bruges et Granada, Convention pour la protection du patrimoine naturel et culturel). En général, il y a une approche commune du patrimoine naturel et bâti voir la Convention pour la protection du patrimoine naturel et culturel, les conventions sur le paysage (Florence, La Valetta et Faro) et l'Agenda 21 (Petrişor, 2011a, b).

Le Tableau 1 pressente une comparaison entre les principes qui composent le fondement des politiques de la conservation des aires naturels et de la biodiversité et leur application pour l'espace bâti.

Tableau 1 : Comparaison entre les principes de la conservation de la biodiversité et de l'espace

\begin{tabular}{|c|c|c|c|}
\hline Type & Principes & Biodiversité & Espace bâti \\
\hline 1 & $\begin{array}{l}\text { Harmonie entre } \\
\text { conservation et } \\
\text { développement }\end{array}$ & $\begin{array}{l}\text { Réinterpréter la } \\
\text { conservation (voir 2) }\end{array}$ & $\begin{array}{l}\text { Intégration entre les bâtiments } \\
\text { conservés et la nouvelle vie urbaine }\end{array}$ \\
\hline 2 & $\begin{array}{l}\text { Conservation comme } \\
\text { maintenance dans un } \\
\text { état favorable }\end{array}$ & $\begin{array}{l}\text { Activités économiques } \\
\text { permises d'une manière } \\
\text { incrémentale (du point de } \\
\text { vue spatial) }\end{array}$ & $\begin{array}{l}\text { Intégration des fonctions dans le } \\
\text { contexte contemporain }\end{array}$ \\
\hline 3 & Représentativité & $\begin{array}{l}\text { Choix des échantillons } \\
\text { représentatifs pour } \\
\text { l'espace biogéographique }\end{array}$ & $\begin{array}{l}\text { Représentativité historique, valeur } \\
\text { culturelle }\end{array}$ \\
\hline 4 & Zonage interne & $\begin{array}{l}\text { Permissions graduelles } \\
\text { (du point de vue spatial) }\end{array}$ & $\begin{array}{l}\text { Limite de protection pour les } \\
\text { monuments, restrictions imposées } \\
\text { aux constructions adjacentes par les } \\
\text { règles d'urbanisme }\end{array}$ \\
\hline 5 & $\begin{array}{l}\text { Assurer le support } \\
\text { des populations } \\
\text { locales }\end{array}$ & $\begin{array}{l}\text { Implication de la } \\
\text { population locale dans le } \\
\text { processus d'élaborer le } \\
\text { plan de management }\end{array}$ & $\begin{array}{l}\text { Après la restauration, les bâtiments } \\
\text { historiques deviennent centres } \\
\text { d'attraction pour les activités } \\
\text { communautaires ; intégration dans } \\
\text { la vie de la ville (jour et nuit) }\end{array}$ \\
\hline 6 & $\begin{array}{l}\text { Coopération } \\
\text { nationale et } \\
\text { internationale }\end{array}$ & $\begin{array}{l}\text { Formation des réseaux } \\
\text { des aires naturelles } \\
\text { protégées }\end{array}$ & $\begin{array}{l}\text { Conventions internationales } \\
\text { concernant les monuments } \\
\text { historiques et leur protection }\end{array}$ \\
\hline
\end{tabular}




\section{Conclusion}

L'idée centrale de l'article est que le soutien de la population locale (notamment des propriétaires et locataires des immeubles, mais aussi de la communauté locale) est très important pour la conservation. Dans ce contexte, les principes de la conservation biologique - conservation fonctionnelle, gestion différentiée, représentativité, soutien local et international, zonage interne, coopération nationale et internationale - sont également applicables à la conservation du patrimoine architectural.

\section{Références}

Berkes F., Floke C., Gadgil M., 1995. Traditional ecological knowledge, biodiversity, resilience and sustainability, dans: Perrings C. A., Mäler K.-G., Folke C., Holling C. S., Jansson B.-O. (Editeurs), Biodiversity Conservation, Kluwer, Dordrechts, Pays-Bas, p. 281-300.

Bouroumi M. T., Beghdoud L. K., Hamma W., 2017. The Algerian coastline between degradation and protection of the heritage: The case of the coastal town of Ain El Turck. Urbanism Architecture Constructions, 8, 3, 249-264, URBAN-INCERC, Bucarest, Roumanie.

Brundtland G. H., 1987. Our Common Future. WCED, Oxford University Press, Oxford, Grande Bretagne.

Cercleux A.-L., Merciu F.-C., Merciu G.-L., 2012. Models of technical and industrial heritage reuse in Romania. Procedia Environmental Sciences. 14. 216-225.

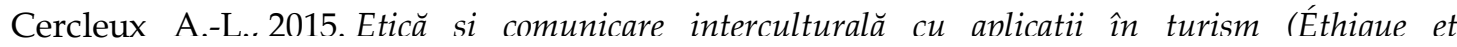
communication interculturelle avec des applications en tourisme). Presses universitaires, Bucarest, Roumanie.

Cercleux A.-L., Merciu C., Merciu G.-L., 2016. A model of development strategy encompassing creative industries to reduce visual pollution - Case study: Strada Franceză, Bucharest's old city. Procedia Environmental Sciences, 32, 404-411.

CHCfE Consortium, 2015. Cultural heritage counts for Europe. International Cultural Centre, Cracovie, Pologne.

Collignon P., 2009. Situation et défis du monde rural - Le patrimoine et le paysage au cour des enjeux du développement territorial durable, Aménagement du territoire européen et paysage 88: 99-103.

de la Torre M., Mason R., 2002. Introduction, dans: de la Torre M. (Éditeur), Assessing the values of cultural heritage research report, The Getty Conservation Institute, Los Angeles, Californie, États-unis, p. 3-4.

DYNAMO Transnational Group, 2006. Espon Project 1.3.3. DYNAMO, The role and spatial effects of cultural heritage and identity. Mapping the Dynamics of European Culture: Pressure and Opportunities from the European Enlargement, Report. INTERREG III - ESPON, Luxembourg, Luxembourg.

Elsorady E. D., 2014. Assessment of the compatibility of new uses for heritage buildings: the example of Alexandria National museum, Alexandria, Egypt. Journal of Cultural Heritage, 15, 5, 511-521, Elsevier, Amsterdam, Pays-Bas.

European Commission, (2015) Getting cultural heritage to work for Europe Report of the Horizon 2020 Expert Group on Cultural Heritage. http://ec.europa.eu/assets/eac/culture/library/studies/ccs-feasibility-study_en.pdf

Ferretti V., Bottero M., Mondini G., 2014. Decision-making and cultural heritage: an application of the multi-attribute value theory for the reuse of historical buildings. Journal of Cultural Heritage, 15, 6, 644-655, Elsevier, Amsterdam, Pays-Bas. 
Frey B., 1997. The evaluation of cultural heritage: Some critical issues, dans: Hutter M., Rizzo I. (Éditeurs), Economic Perspectives on Cultural Heritage, Macmillan, Londres, Royaume Uni, p. 31-49.

Grasso S., Maugeri M., 2009. The road map for seismic risk analysis in a Mediterranean city. Soil Dynamics and Earthquake Engineering, 29, 1034-1045, Elsevier, Amsterdam, PaysBas.

Graz C., 2011. Réflexions autour des inventaires, manuels et méthodes: un certain nombre d'outils opérationnels exemplaires développés dans le cadre des projets Euromed Heritage méritent d'être introduits, diffuses et adaptés, y compris au-delà du champ méditerranéen. ICOMOS Atellers - débats, Session 1: Le patrimoine, moteur de développement, p. 996-998.

Greffe X., 2011. L'économie politique du patrimoine culturel. ICOMOS, Atellers - débats, Session 1: Le patrimoine, moteur de développement, p. 928-936.

Hamma W., 2017a. Classification and recognition of the heritage values of the monuments of Tlemcen. Urbanism Architecture Constructions, 8, 2, 129-142, URBAN-INCERC, Bucarest, Roumanie.

Hamma W., 2017b. Method of rehabilitation of old urban fabrics of Tlemcen. Urbanism Architecture Constructions, 8, 4, 315-330, URBAN-INCERC, Bucarest, Roumanie.

Hamma W., Djedid A., Ouissi M. N., 2016. Intervention on historical city Tlemcen during 19602009: impacts and applicability. Urbanism Architecture Constructions, 7, 2, 109-124, URBAN-INCERC, Bucarest, Roumanie.

Haspel J., 2011. Built heritage as a positive location factor-economic potentials of listed properties. ICOMOS Atellers - débats, Session 1: Le patrimoine, moteur de development, p. 905916.

Heritage A., Golfomitsou S., 2015. Conservation science: Reflections and future perspectives. Studies in Conservation, 60, sup. 2, 2-6, ICCROM, Rome, Italie.

Hoag D. L., Skold M. D., 1996. The relationship between conservation and sustainability. Journal of Soil and Water Conservation 51, 4292-4295, Soil and Water Conservation Society, Ankeny, IA, États-unis.

Holling C. S., 2000. Theories for sustainable futures. Conservation Ecology, 4, 2, 7, Resilience Alliance, Wolfville, Nova Scotia, Canada.

ICOMOS, (2009). ICOMOS Report World Heritage in Danger Compendium II ( A compendium of key decisions on the conservation of cultural heritage properties on the UNESCO List of World Heritage in Danger), 59 p., file:///C:/Users/georg/Downloads/event-740-11.pdf

Lacaze J.-P., 1990. Les méthodes de l'urbanisme. 2éme édition corrigée. Presses Universitaires de France, Paris, France, $127 \mathrm{p}$.

Maroevic I., 1998. The phenomenon of cultural heritage and the definition of a unit of material. Nordisk Museologi, 2, 135-142, Université d'Umeå, Umeå, Suède.

Marques J. C., 2001. Diversity, Biodiversity, Conservation, and Sustainability, The Scientific World, 1, 534-543, Hindawi, Nasr City, Caire, Égypte.

Mason R., 2002. Assessing values in conservation planning: methodological issues and choices, dans: de la Torre M. (Éditeur), Assessing the values of cultural heritage research report, The Getty Conservation Institute, Los Angeles, Californie, États-unis, p. 5-30.

Merciu C., Merciu G.-L., Paraschiv M., 2014. Industrial heritage assessment in the light of the cultural regeneration: challenges, opportunities and solutions for reuse. Annals of Professional Association of Geographers of Romania, 5, 5: 5-14, Association Professionnelle des Géographes Roumains, Bucarest, Roumanie.

Merciu F.-C., Cercleux A. L., Merciu G., 2016. L'évaluation des bâtiments de patrimoine dans la perspective de la reconversion fonctionnelle. Étude de cas: la Rue Lipscani, Centre Historique de Bucarest. Annals of the University of Bucharest, Geography Series, 65, 55-62, Université de Bucarest, Bucarest, Roumanie.

Nasser N., 2003. Planning for Urban Heritage Places: Reconciling Conservation, Tourism, and Sustainable Development. Journal of Planning Literature, 17, 4, 467-479, Sage, Thousand Oaks, CA, Etats-Unis. 
Naveh Z., 1994. From Biodiversity to ecodiversity: a landscape - ecology approach to conservation and restoration. Restoration Ecology, 2, 3, 180-189, John Wiley \& Sons Inc., Malden, MA, États-unis.

Naveh Z., 1998. Ecological and cultural landscape restoration and the cultural evolution towards a post-industrial symbiosis between human society and nature. Restoration Ecology, 6, 2, 135-143, John Wiley \& Sons Inc., Malden, MA, États-unis.

Nelson H., Vertinsky I. , 2005. The International Trade and Environmental Regime and the Sustainable Management of Canadian Forests, dans: Kant S., Berry R. A. (Éditeurs), Institutions, Sustainability, and Natural Resources Institutions for Sustainable Forest Management, Springer, Amsterdam, Pays-Bas, p. 257-296.

Nocca F., 2017. The role of cultural heritage in sustainable development: multidimensional indicators as decision-making tool. Sustainability, 9, 1-28, MDPI AG, Basel, Suisse.

Pagnini L., Vicente R., Lagomarsino S., Varum H., 2011. A mechanical model for the seismic vulnerability assessment of old masonry buildings. Earthquake and Structures, 2, 1, 25-42, Techno Press, Daejeon, Corée.

Pessina V., Meroni F., 2009. A WebGis tool for seismic hazard scenarios and risk analysis. Soil Dynamics and Earthquake Engineering, 29, 9, 1274-1281, Elsevier, Amsterdam, PaysBas..

Petrişor A.-I., 2011a. Capitalul cultural, vector al dezvoltării durabile (Le capital culturel, vecteur du développement durable), dans : Sârbu C. N. (Éditeur), Peisaj cultural şi dezvoltare (Paysage culturel et développement), Presses de l'Université d'architecture et d'urbanisme "Ion Mincu », Bucarest, Roumanie, p. 223-232.

Petrişor A.-I., 2011b. Patrimoniul natural şi cultural: o abordare integrată reflectată în convenții internationale (Le patrimoine naturel et culturel : une démarche intégrée réfléchie par les conventions internationales), Repere, 7, 84-87, Éditions Alfa, Iaşi, Roumanie.

Petrişor A.-I., 2011c. Principii spațiale ale conservării biodiversității prin arii naturale protejate (Principes spatiaux de conservation de la biodiversité par les aires naturelles protégées). Analele Arhitecturii, 6, 1, 37-39, Presses de l'Université d'architecture et d'urbanisme « Ion Mincu », Bucarest, Roumanie.

Petrişor A.-I., (2013), Multi-, trans- and inter-disciplinarity, essential conditions for the sustainable development of human habitat, Urbanism Architecture Constructions 4, 2, 43-50, URBAN-INCERC, Bucarest, Roumanie.

Petrişor A.-I., Petrişor L. E., 2014. 25 years of sustainability. A critical analysis. Present Environment and Sustainable Development 8, 1, 175-190, Presses de l'Université « Alexandru Ioan Cuza » de Iaşi, Roumanie.

Rodwell D., 1992. Preserving and Enhancing. Context, 36, 16, Institute of Historic Building Conservation, Stafford Road, Tunbridge Wells, Kent, Écosse.

Rodwell, D., 2003. Sustainability and the Holistic Approach to the Conservation of Historic Cities. Journal of Architectural Conservation, 9(1), 58-73, Taylor \& Francis, New York, NY, Etats-Unis.

Rodwell, D., 2007. Conservation and sustainability in historic cities. Blackwell Publishing, Londres, Royaume Uni.

Ruijgrok E. C. M., 2006. The three economic values of cultural heritage: a case study in the Netherlands. Journal of Cultural Heritage, 7, 3, 206-213, Elsevier, Amsterdam, PaysBas.

Sabbioni C., Cassar M., Brimblecombe P., Lefevre R. A., 2008. Vulnerability of Cultural Heritage to Climate Change Report, European and Mediterranean Major Hazards Agreement (EUR-OPA), Conseil de l'Europe, Strasbourg, France.

Sandu I. C. A., Spiridon P., Sandu I., 2016. Current studies and approaches in the field of cultural heritage conservation science. Harmonising the terminology in an interdisciplinary context. International Journal of Conservation Science, 7(3), 591-606, "Alexandru Ioan Cuza" University Publishing House, Iaşi, Romania.

Sandu I., 2010. Editorial. International Journal of Conservation Science, 1(1), 1-1, “Alexandru Ioan Cuza" University Publishing House, Iaşi, Romania. 
Stephenson V., D'Ayala D., 2014, A new approach to flood vulnerability assessment for historic buildings in England. Natural Hazards and Earth System Sciences, 14, 1035-1048, Copernicus Publications, Göttingen, Allemagne.

Throsby D., 2002. Cultural capital and sustainable concepts in the economics of cultural heritage, dans: de la Torre M. (Éditeur), Assessing the values of cultural heritage research report, The Getty Conservation Institute, Los Angeles, Californie, Etats-Unis, p. 101-117.

UNESCO (United Nations Educational, Scientific, and Cultural Organization), 2008. Policy Document on the Impacts of Climate Change on World Heritage Properties, Paris, France, 14

UNESCO (United Nations Educational, Scientific, and Cultural Organization), 2014. Cultural for development indicators. Methodology manual. Paris, France, $142 \mathrm{p}$.

Vecco M., 2010. A definition of cultural heritage: from the tangible to the intangible. Journal of Cultural Heritage, 11, 3, 321-324.

Vicente R., Varum H., Mendes da Silva, J. A. R., 2006. Vulnerability assessment of traditional buildings in Coimbra, Portugal, supported by a GIS tool. Proceedings of the Conference on Earthquake Engineering and Seismology, p. 1-10. 